\title{
Técnicas alternativas del manejo de la conducta en odontopediatría
}

\section{Resumen}

Diversas técnicas convencionales han sido utilizadas con gran eficacia; sin embargo, la no aceptación de algunas de ellas por los padres, ciertos condicionamientos éticos y legales así como cambios en la educación actual y el avance en nuevas tecnologías, obligan al odontólogo a un replanteamiento de actitudes. Objetivo: El objetivo del presente trabajo es identificar qué técnicas alternativas son empleadas en la clínica dental por los profesionales para el manejo de la conducta de pacientes infantiles. Material y métodos: Se realizó un cuestionario en la plataforma de Google, remitido vía email a 190 odonto/ estomatólogos que tratasen pacientes infantiles, obteniendo una participación de 132 encuestas adecuadas para el análisis de 6 técnicas alternativas de manejo de la conducta. El estudio estadístico se realizó en el programa SPSS aplicando el test de ANOVA para una significación de $\mathrm{p}=0,05$.

\author{
Begoña Bartolomé Villar, ${ }^{1}$ \\ Mercedes Méndez Zunino, ${ }^{2}$ \\ Cristina Vilar Rodríguez, ${ }^{2}$ \\ Juan José Arrieta Blanco. ${ }^{4}$
}

Resultados: Un 35,1\% de los encuestados utilizaban medios audiovisuales para el tratamiento dental, siendo la televisión el más frecuentemente empleado. La musicoterapia fue la técnica más utilizada (70,5\% de los participantes) con mayor frecuencia del sexo femenino, aunque sin diferencias significativas, siendo la música moderna la de mayor aceptación. Un 32,8\% utilizaron la ludoterapia como técnica de manejo seguida del empleo de técnicas de relajación con un $25 \%$, ambas con mayor porcentaje femenino. Las dos técnicas menos utilizadas fueron la aromaterapia $(6,8 \%)$ y la hipnosis $(2,3 \%)$. Conclusiones: A pesar del incremento en la utilización de técnicas alternativas en el manejo de la conducta, todavía su empleo es escaso por los odonto/estomatólogos.

Palabras clave: Manejo de conducta en odontopediatría; ansiedad dental, odontopediatría.

\footnotetext{
${ }^{1}$ Profesora Titular de Odontopediatría. Universidad Europea de Madrid, Facultad de Ciencias Biomédicas, Departamento de Odontología. Madrid, España. ${ }^{2}$ Profesora Adjunta de Odontopediatría. Universidad Europea de Madrid, Facultad de Ciencias Biomédicas, Departamento de Odontología. Madrid, España. ${ }^{3}$ Profesora Adjunta de Odontopediatría. Universidad Europea de Madrid, Facultad de Ciencias Biomédicas, Departamento de Odontología. Madrid, España. ${ }^{4} J e f e$ Asociado del Servicio de Estomatología. Hospital Universitario Fundación Jiménez Díaz, Servicio de Estomatología-Odontología. Madrid, España.
} 
Artigo original

\section{Técnicas alternativas para a gestão comportamental em odontologia pediátrica}

\section{Resumo}

Várias técnicas convencionais têm sido usadas com grande eficácia; contudo, a não aceitação de algumas delas pelos pais, certas condições éticas e legais, assim como as mudanças na educação atual e o avanço das novas tecnologias, forçam o dentista a repensar as atitudes. Objetivo: O objetivo do presente estudo é identificar quais técnicas alternativas são utilizadas na clínica odontológica por profissionais para gerenciar o comportamento de pacientes infantis. Material e métodos: Foi realizado um questionário na plataforma Google, enviado por e-mail a 190 dentistas/ stomatologistas que trataram crianças, obtendo 132 pesquisas adequadas para a análise de 6 técnicas alternativas de gestão comportamental. $\mathrm{O}$ estudo estatístico foi realizado no programa SPSS aplicando o teste ANOVA para uma significância de $p=0,05$. Resultados: $35,1 \%$ dos inquiridos utilizaram meios audiovisuais para tratamento dentário, sendo a televisão a mais frequentemente utilizada. A musicoterapia foi a técnica mais utilizada (70,5\% dos participantes) pelo sexo feminino, embora sem diferenças significativas, sendo a música moderna a mais amplamente aceita. 32,8\% utilizaram a terapia lúdica como técnica de gestão seguida do uso de técnicas de relaxamento com $25 \%$, ambas com uma percentagem maior de mulheres. As duas técnicas menos utilizadas foram a aromaterapia $(6,8 \%)$ e a hipnose (2,3\%). Conclusões: Apesar do aumento do uso de técnicas alternativas na gestão do comportamento, o seu uso ainda é escasso pelos odontologistas/ estomatologistas.

Palavras-Chave: Gestão comportamental em odontologia pediátrica; ansiedade dentária, odontologia pediátrica.

Original article

\section{Alternative behavioral management techniques in pediatric dentistry}

\begin{abstract}
Various conventional techniques have been used with great efficiency; nevertheless, the non-acceptance of some of them by the parents, certain ethical and legal conditions as well as changes in the current education and the advance in new technologies, force the dentist to a
\end{abstract}

rethinking of attitudes. Aim: The aim of this paper is to identify what alternative techniques are used by professionals for the behavior's management of pediatric patients in the dental clinic. Material and methods: A questionnaire was carried out on the Google platform, sent via email to 190 odonto / stomatologists who treated pediatric patients, obtaining a 
participation of 132 appropriate surveys for the analysis of 6 alternative behavior management techniques. The statistical study was carried out in the SPSS program, applying the ANOVA test for a significance of $p=0.05$. Results: $35.1 \%$ of respondents used audiovisual media for dental treatment, being television the most frequently used. Music therapy was the most applied technique $(70.5 \%$ of the participants) with the highest frequency of female sex, without significant differences though, being modern music the most

\section{Introducción}

Diversos métodos convencionales han sido empleados para disminuir la ansiedad y miedo dental del paciente infantil; sin embargo, el rechazo de los padres ante algunas técnicas más agresivas consideradas como potencialmente traumáticas, ciertas consideraciones éticas y legales así como cambios socioculturales en la educación, obligan a un replanteamiento de actitudes. Todo ello junto al gran avance de recursos tecnológicos, el cambio en la conducta pediátrica con menor autocontrol y la preocupación por el empleo de métodos no farmacológicos, hace que el odontólogo se eduque en otras técnicas alternativas, incorporándolas a su práctica clínica ${ }^{1}$ con el fin de mejorar la comunicación con el paciente infantil. Entre ellas cabría destacar:

- Aromaterapia: La utilización de determinados olores puede promover efectos positivos, tanto a nivel físico como emocional, mediante la liberación widely accepted. $32.8 \%$ used play therapy as a management technique followed by the use of relaxation techniques with $25 \%$, both with a higher female percentage. The two least used techniques were aromatherapy $(6.8 \%)$ and hypnosis $(2.3 \%)$. Conclusions: Despite the increase in the use of alternative techniques in behavior management, their use is still scarce by dentists / stomatologists.

Key Words: Behavior management in pediatric dentistry; dental anxiety; pediatric dentistry.

de neurotransmisores que inducen relajación. Se han observado cambios en el nivel de ansiedad, frecuencia cardiaca y respiratoria. ${ }^{2,3}$

- Medios audiovisuales: La tecnología permite utilizar diversos medios de distracción proporcionando al paciente una evasión de estímulos auditivos $\mathrm{y}$ visuales externos que pueden ser desagradables, fundamentalmente tras administración de anestesia local. Estos medios pueden ser empleados a modo de videos modelo donde se expliquen los procedimientos previos a la visita dental. Diversos estudios ${ }^{4,5}$ han demostrado su eficacia al mejorar el nivel de ansiedad; si bien otros no han observado diferencias significativas aunque admiten una mejora en el comportamiento. ${ }^{6}$ Liu et al. concluyen que existe una baja evidencia de los efectos beneficiosos de la distracción visual para reducir la ansiedad, aunque parece mejorar el comportamiento en niños con ansiedad leve/moderada. ${ }^{7}$ 
- Musicoterapia: El empleo de la música supone una técnica útil y fácil de usar, disminuyendo el stress no sólo en pacientes sino incluso en los propios profesionales. ${ }^{8}$ Para la obtención de un efecto positivo parece importante el tipo de música utilizado; así, un estudio llevado a cabo sobre 150 niños indicó que las historias auditivas tenían mayor efecto que la música de niños y de películas. ${ }^{9}$ Existen trabajos que demuestran su eficacia ${ }^{9,10}$ tanto de forma activa como pasiva. Una revisión sistemática de Moola et al. recomienda, con grado B de evidencia, su utilidad tanto en niños como en adultos siendo preferible a los tratamientos farmacológicos. ${ }^{11}$

- Hipnosis: La hipnosis se utiliza en Odontología para inducir a la relajación, desviando la atención del paciente de los estímulos externos. ${ }^{4}$ A pesar de haberse demostrado su eficacia, este método es poco utilizado ya que exige un cierto entrenamiento por parte del profesional.

- Relajación: Esta técnica, fundamentalmente a través de ejercicios respiratorios, ha sido empleada para reducir la ansiedad con resultados positivos; sin embargo, mientras es admitido que la relajación respiratoria puede ser efectiva en reducir la percepción del dolor, la evidencia es más equívoca respecto a la reducción de la ansiedad. ${ }^{4}$

- Ludoterapia: Constituye una técnica de modificación de la conducta simple, no costosa, no invasiva que puede ser aplicada en cualquier momento de la práctica dental. ${ }^{12}$ Puede abarcar diferentes modalidades desde juegos con materiales físicos a preguntas sobre experiencias agradables o empleo de la imaginación mediante historias. ${ }^{4}$

Dichas técnicas alternativas pueden ser coadyuvantes, junto a las técnicas convencionales, para un adecuado manejo del comportamiento. El objetivo del presente trabajo es identificar qué técnicas alternativas son empleadas en la clínica dental por los profesionales para mejorar la conducta del paciente infantil.

\section{Material y métodos}

Se realizó un estudio observacional, transversal y retrospectivo en la Universidad Europea de Madrid a través de la elaboración de un cuestionario en la plataforma de Google. Dicho cuestionario fue remitido vía email a 190 odonto/estomatólogos obteniendo una participación final de 132 profesionales de manera voluntaria y anónima $(69,47 \%$ de participación).

Criterios de selección de la muestra: Se incluyeron odonto/estomatólogos que dentro de su actividad profesional tratasen pacientes infantiles y que perteneciesen a diferentes especialidades odontológicas.

Variables analizadas: En el cuestionario se recogían datos de filiación respecto a la edad, sexo y actividad profesional principal y se ofrecían preguntas relacionadas con factores ambientales de la consulta: existencia de sala de espera infantil, decoración del gabinete dental y vestuario del profesional, y otras relacionadas con la utilización de 6 técnicas no convencionales para el manejo del comportamiento: medios audiovisuales, 
musicoterapia, aromaterapia, ludoterapia, hipnosis y técnicas de relajación.

El Proyecto fue aprobado por el Comité de Ética de la Investigación de la Universidad Europea de Madrid con el código CIPI/19/030 en Febrero de 2019, cumpliendo los principios éticos de la Declaración de Helsinki. A todos los participantes se les informaba sobre la finalidad de la encuesta, solicitando su consentimiento informado.

Procesamiento de la información: Los datos de las diferentes variables del cuestionario fueron analizados tras ser incluidos en una hoja Excel otorgando códigos cuantificables para el posterior análisis estadístico. Éste se llevó a cabo mediante el programa SPSS realizándose una comparación de medias entre las diferentes técnicas y aplicando el test de ANOVA para determinar la significación $(\mathrm{p}<0,05)$.

\section{Resultados}

Para el análisis de los resultados distribuimos la variable edad y profesión en varios grupos (Tabla 1).

Los dos últimos grupos de especialidades (periodoncia y prostodoncia) fueron agrupados para la elaboración de los resultados dado el bajo porcentaje de representación.

De los 132 cuestionarios, 31 correspondían a varones $(23,48 \%)$ y 101 a mujeres $(76,52 \%)$. Un $47 \%$ de los encuestados tenían en su consulta una sala de espera diseñada para niños; de ellos un $41,9 \%$ correspondían a varones y un $48,5 \%$ a mujeres, no siendo estas diferencias estadísticamente significativas $(p=0,332)$. Analizando por edad, encontramos que los profesionales entre 25-35 años eran los que con mayor frecuencia diseñaban en sus consultas una zona de espera infantil

Tabla 1: Distribución por edad y especialidad de los profesionales.

\begin{tabular}{|c|c|}
\hline Profesionales & N (Porcentaje) \\
\hline Menores de 25 años & $10(7,6 \%)$ \\
\hline Entre 25-35 años & $66(50 \%)$ \\
\hline Entre 36-45 años & $32(24,2 \%)$ \\
\hline Entre 46-55 años & $9(8 \%)$ \\
\hline Mayores de 55 años & $15(11,4 \%)$ \\
\hline Odontología General & $61(46,2 \%)$ \\
\hline Odontopediatría & $24(18,2 \%)$ \\
\hline Ortodoncia & $15(11,4 \%)$ \\
\hline Cirugía & $12(9,1 \%)$ \\
\hline Endodoncia & $11(8,35 \%)$ \\
\hline Periodoncia & $5(3,8 \%)$ \\
\hline Prostodoncia & $4(3 \%)$ \\
\hline
\end{tabular}


Tabla 2: Vestuario del profesional: Distribución por sexos.

\begin{tabular}{|l|c|c|c|c|}
\hline & Varón & Mujer & Total & Significación \\
\hline Pijama color liso & $15(48,4 \%)$ & $21(20,8 \%)$ & $36(27,3 \%)$ & 0,001 \\
\hline Pijama blanco & $15(48,4 \%)$ & $50(49,5 \%)$ & $65(49,2 \%)$ & 0,000 \\
\hline Pijama infantil & $1(3,2 \%)$ & $30(29,7 \%)$ & $31(23,5 \%)$ & 0,000 \\
\hline Total & $31(100 \%)$ & $101(100 \%)$ & $132(100 \%)$ & \\
\hline
\end{tabular}

y los mayores de 55 años los que menos; disponiendo de ella especialmente los ortodoncistas $(73,3 \%)$ seguidos de los odontopediatras (58,3\%).

Un 53\% de los encuestados refirió tener algún tipo de decoración con motivos infantiles en el gabinete dental; de ellos, las odontólogas tenían mayor preferencia frente a los varones $(57,4 \%$ / 38,7 \% respectivamente), con diferencias casi significativas $(p=0,052)$. Al analizar por edad, encontramos que el grupo entre 46-55 años son los que más decoran sus gabinetes $(66,7 \%)$ y por especialidad son los odontopediatras $(79,2 \%)$, existiendo diferencias significativas respecto al resto de profesionales $(p=0,026)$.

En relación al vestuario se encontró que tan sólo un 23,5\% tenían pijama con motivos infantiles, con clara predisposición hacia las mujeres (diferencias estadísticamente significativas) (Tabla 2), odontopediatras y ortodoncistas.
Un 35,1\% de los encuestados utilizaban medios audiovisuales para el tratamiento dental; de ellos un 41,9 \% eran varones y un $33 \%$ mujeres, no siendo estas diferencias significativas $(p=0,362)$. Los profesionales entre 46-55 años y los odontopediatras eran los que más los empleaban. La televisión fue el medio audiovisual más utilizado y el que menos las gafas de video, no existiendo diferencias significativas entre ellos $(p=0,616)$. La distribución delos diferentes medios por sexos la observamos en la Tabla 3.

La música fue utilizada por el 70,5\% de los participantes con mayor frecuencia del sexo femenino $(73,3 \% / 61,3 \%)$ aunque sin diferencias significativas $(p=0,146)$. La música moderna fue la de mayor aceptación (Tabla 4), siendo los profesionales entre 25-35 años los que más la empleaban en las diferentes modalidades.

Tabla 3: Distribución de los medios audiovisuales empleados por sexos.

\begin{tabular}{|l|c|c|c|}
\hline & Varón & Mujer & Total \\
\hline Televisión (porcentaje dentro del sexo) & $5(38,5 \%)$ & $11(33,3 \%)$ & $16(34,8 \%)$ \\
\hline Tablet & $4(30,8 \%)$ & $6(18,2 \%)$ & $10(21,7 \%)$ \\
\hline Smartphones & $3(23,1 \%)$ & $9(27,3 \%)$ & $12(26,1 \%)$ \\
\hline Gafas & $1(7,7 \%)$ & $7(21,2 \%)$ & $8(17,4 \%)$ \\
\hline Total & $13(100 \%)$ & $33(100 \%)$ & $46(100 \%)$ \\
\hline
\end{tabular}


Tabla 4: Distribución del tipo de música por sexos.

\begin{tabular}{|l|c|c|c|}
\hline & Varón & Mujer & Total \\
\hline Infantil & $2(10,5 \%)$ & $17(23,0 \%)$ & $19(20,4 \%)$ \\
\hline Clásica & $5(26,3 \%)$ & $21(28,4 \%)$ & $26(28,0 \%)$ \\
\hline Moderna & $12(63,2 \%)$ & $36(48,6 \%)$ & $48(51,6 \%)$ \\
\hline Total & $19(100 \%)$ & $74(100 \%)$ & $93(100 \%)$ \\
\hline
\end{tabular}

Un 32,8 \% de los profesionales encuestados emplearon la ludoterapia como técnica de manejo, con mayor frecuencia de las mujeres (35\%/ 25,8\%). Los odontólogos mayores de 55 años (53,3 \%) la utilizaban más que los jóvenes (tan sólo un 10\% pertenecían al grupo menor de 25 años). En relación a la dedicación principal de su actividad no observamos diferencias significativas $(p=0,127)$ si bien los odontopediatras $(50 \%)$ seguidos de los ortodoncistas $(40 \%)$ son los que más realizaban juegos a la hora de trabajar con los pacientes infantiles.

Tan sólo un 6,8 \% de los profesionales usaban aromaterapia en su consulta dental, con prácticamente igual proporción de hombres y mujeres $(6,5 \%$ / 6,9 \% respectivamente), no existiendo diferencias significativas por sexos $(p=0,926)$ ni por grupos de edad ni en las diferentes especialidades analizadas. Los aromas más utilizados fueron lavanda y manzanilla.

En relación a la hipnosis, tan sólo un 2,3\% de los encuestados refirió emplearla para manejar la conducta, fundamentalmente varones $(6,5 \% / 1 \%)$, menores de 25 años y cirujanos, no existiendo diferencias significativas ni en cuanto a la edad $(p=0,475)$ ni entre las diferentes especialidades $(p=0,603)$.
Tampoco existieron diferencias respecto al sexo en cuanto al uso de técnicas de relajación, sobre todo a través de ejercicios respiratorios $(p=0,407)$, siendo empleadas por un $25 \%$ de los profesionales, con ligera predominancia del sexo femenino $(26,7 \%$ mujeres / 19,4\% varones). Los dentistas de 46-55 años fueron los que más las aplicaban $(44,4 \%)$, no existiendo una predilección especial por ninguna especialidad.

\section{Discusión}

El hecho de enfrentarnos hoy día con una sociedad más permisiva y a la vez más demandante, obliga al odontólogo a abandonar ciertas técnicas de manejo del comportamiento más restrictivas e intentar utilizar otras de mayor aceptación para los padres y para el paciente infantil, tanto desde el punto de vista físico como psicológico. Así, un estudio llevado a cabo por Muhammad et al. encuentra que la distracción fue una técnica tan bien aceptada como decir- mostrarhacer $(99,2 \%)$ mientras que la hipnosis y separación de los padres fueron sólo moderadamente aceptadas. ${ }^{13}$ Alammouri concluye que tanto el refuerzo positivo como decir-mostrar-hacer y la distracción son admitidas por los padres con más de un $75 \%$ de aceptación. ${ }^{14}$ Frente a ello, algunos autores señalan la preferencia por técnicas de manejo tradicionales sobre aquellas que utilizan nueva tecnología, siendo un poco reacios a su incorporación, fundamentalmente por el coste, esfuerzo y sobre todo el tiempo que conllevan. ${ }^{15}$

Ya Wright et al., en 1991, señalan cómo una de las técnicas más empleadas 
para disminuir la ansiedad del paciente infantil es tener áreas en la sala de espera con mobiliario y materiales infantiles. ${ }^{16}$ En nuestros resultados resaltamos que prácticamente la mitad de odontólogos que trabajan con niños tienen una sala de espera especialmente diseñada y/o algún tipo de decoración infantil en el gabinete. Esta cifra es bastante cercana al 55,5\% de Walli et al. en una encuesta realizada a cirujanos dentales. ${ }^{17}$

La musicoterapia, la realidad virtual y los recursos audiovisuales son medios no farmacológicos frecuentemente empleados para disminuir la ansiedad y miedo en el paciente infantil, siendo la musicoterapia la técnica más fácil de emplear ya que otros métodos son más costosos. Una revisión sistemática sobre 21 estudios considera que las diversas técnicas de distracción son efectivas, aunque con baja evidencia científica, si bien pueden constituir una buena estrategia ya que en la mayoría de los estudios las diferencias son significativas. ${ }^{18}$ De ahí que un trabajo realizado en estudiantes sobre diversas técnicas a lo largo de su formación, demuestre que la distracción es una de las técnicas más aceptadas por alumnos pregraduados ${ }^{19}$. Por ello, algunos autores abogan que las técnicas de conducta cognitiva como refuerzo positivo, distracción, desensibilización y relajación deben ser un estándar en el tratamiento, debiendo ser incluidas en los programas dentales. ${ }^{20}$

En nuestro estudio encontramos que tan sólo un $35,1 \%$ de los encuestados utilizaban algún medio de distracción audiovisual, siendo el más empleado la televisión seguido de las tablets. Esta cifra está cercana al 33,5\% de la encuesta realizada a cirujanos pakistaníes ${ }^{17}$ pero muy alejada del $61 \%$ de la aplicada a cirujanos maxilofaciales turcos, ${ }^{21}$ del $86,5 \%$ de profesionales de Tanzania ${ }^{22}$ o del $89,7 \%$ de estudiantes estadounidenses. ${ }^{20}$ Estas diferencias pueden deberse a que los trabajos citados hablan sobre medidas de distracción de forma generalizada, por lo que pueden englobar varias de ellas, siendo desglosadas en nuestro estudio. Otros artículos, sin embargo, reflejan cómo la mayoría de participantes (89 \%) no utilizaban estrategias como televisión, video, audios o hipnosis achacando la insuficiente información y educación sobre ellas. ${ }^{16}$

Los odontopediatras fueron los profesionales que más emplearon este medio de distracción, hecho señalado también por Wells et al. ${ }^{23}$, siendo además los que utilizan un mayor y más variado número de técnicas respecto a los dentistas generales, sobre todo aquellas más especializadas y sofisticadas. ${ }^{24}$

La música fue el medio más empleado en nuestro ámbito $(70,5 \%$ de los participantes), fundamentalmente la música moderna, cifra superior al estudio de Levy y Domoto donde el $50 \%$ de los dentistas utilizaban auriculares durante el tratamiento. ${ }^{25}$ No observamos predilección por ninguna especialidad; sin embargo, miembros de la Sociedad Australiana de Odontopediatría encuentran un mayor empleo de la musicoterapia por estos profesionales respecto a los dentistas generales. $^{26}$

Las técnicas de relajación fueron empleadas por el $25 \%$ de los odontólogos; resultados más elevados $(53 \%)$ son reflejados por Levy y Domoto. ${ }^{25}$ Strom et al. encuentran que es la segunda técnica más utilizada 
(35\%), siendo la primera decir-mostrarhacer $(87 \%)$ y la tercera la distracción $(25 \%)$, no estando asociada a la edad ni sexo del odontólogo. ${ }^{27}$

La aplicación del juego como técnica de manejo ha sido un método ampliamente desarrollado. En nuestro estudio encontramos su utilización por el 32,8\% de los odontólogos. Radhakrishna et al. observan cómo la variación de la técnica de decir-mostrar-hacer por decir-mostrarjugar y el empleo de juegos de dentistas explicando los procedimientos a realizar, muestra una mayor efectividad. ${ }^{28}$

La técnica menos empleada en nuestro estudio fue la hipnosis $(2,3 \%)$, cifra similar a otros trabajos, ${ }^{21,29}$ quizá porque su incorporación a la práctica clínica requiere cierto entrenamiento. Peretz et al. la consideran como la técnica menos usada (10\%), ${ }^{30}$ mientras que Wright et al., en una encuesta nacional llevada a cabo en Australia, observan cómo el $83 \%$ de profesionales nunca había utilizado la hipnosis. ${ }^{16}$

\section{Conclusiones}

Dentro de las técnicas consideradas como no convencionales, la más empleada fue la musicoterapia seguida de los medios audiovisuales, ludoterapia, técnicas de relajación, aromaterapia e hipnosis, no encontrando diferencias significativas en relación al sexo. La televisión fue el medio audiovisual más utilizado y la música moderna la más aceptada por los pacientes infantiles, aunque su empleo por los odonto/estomatólogos todavía es escaso.

\section{Referencias bibliográficas}

1. Sheller B. Challenges of managing child behavior in the 21st century dental setting. Pediatr Dent 2004; 26: 111-13.

2. Rashidi F, Jalali M, Karami E, Ebrahimi AR. The effect of music and lavander's aroma on patients anxiety during periodontal surgery. J Dent Mater Tech 2018; 7 (3): 117-122.

3. S PK, Aafaque S, S S, N N. Effect of aromatherapy on dental anxiety among orthodontic patients: A randomized controlled trial. Cureus 2019; 11(8): e5306. doi: 10.7759/cureus.5306.

4. Armfield JM, Heaton LJ. Management of fear and anxiety in the dental clinic: a review. Aust Dent J 2013; 58: 390-407.

5. Zhang C, Qin D, Shen L, Ji P, Wang J. Does audiovisual distraction reduce dental anxiety in children under local anesthesia? A systematic review and meta-analysis. Oral Dis 2019; 25 (2): 416-424.

6. Garrocho-Rangel A, Ibarra-Gutiérrez E, Rosales-Bérber M, Esquivel-Hernández R, Esparza-Villalpando V, Pozos-Guillén A. A video eyeglasses/earphones system as distracting method during dental treatment in children: A crossover randomised and controlled clinical trial. Eur J Paediatr Dent 2018; 19 (1): 74-79.

7. Liu Y, Gu Z, Wang Y, Qi W, Chen V, Xu X, Zhou X. Effect of audiovisual distraction on the management of dental anxiety in children: A systematic review. Int J Paediatr Dent 2019; 29 (1): 14-21.

8. Bancalari L, Oliva P. Efecto de la musicoterapia sobre los niveles de estrés de los usuarios internos de la clínica de Odontología de la Universidad del Desarrollo. Int. J. Odontostomat 2012; 6 (2): 189-193.

9. Navit S, Johri N, Khan SA, Singh RK, Chadha D, Navit P et al. Effectiveness and comparison of various audio distraction aids in management of anxious dental paediatric patients. J Clin Diagn Res 2015; -9 (12): ZC05-ZC09.

10. Mejía-Rubalcava C. Changes induced by music therapy to physiologic parameters in patients with dental anxiety. Complement Ther Clin Pract 2015; 21: 282-86.

11. Moola S, Pearson A, Hagger C. Effectiveness of music interventions on dental anxiety in paediatric and adult patients: a systematic review. JBI Library of Systematic Reviews 2011; 9 (18): 588-630. [citado 201909-19]. .81202008000200004\&lng=es\&nrm=iso>. ISSN 1727-8120. 
12. Dp Kiran S, Vithalani A, J Sharma D, C Patel M, Bhatt R, Srivastava M. Evaluation of the efficacy of play therapy among children undergoing dental procedure through drawings assessed by graphological method: A clinical study. Int J Clin Pediatric Dent 2018; 11 (5): 412-16.

13. Muhammad S, Shyama M, Al-Mutawa SA. Parental attitude toward behavioral management techniques in dental practice with schoolchildren in Kuwait. Med Princ Pract 2011; 20: 350-55.

14. Alammouri M. The Attitude of parents toward behavior management techniques in pediatric dentistry. J Clin Pediatr Dent 2006; 30 (4): 310-13.

15. Allen KD, Stanley RT, McPherson K.. Evaluation of behavior management technology dissemination in pediatric dentistry. Pediatric Dent 1990; 12 (2): 79- 82.

16. Wright FAC, Giebartowski JE, McMurray NE. A national survey of dentists' management of children with anxiety or behaviour problems. Aust Dent J 1991; 36 (5): 378-83.

17. Walli A, Siddiqui TM, Khan R, Batool K. Knowledge, attitude, and practices of dental surgeons in managing child patients. Int J Clin Pediatr Dent 2016; 9 (4): 372-78.

18. Prado IM, Carcavalli L, Abreu LG, Serra-Negra JM, Paiva SM, Martins CC. Use of distraction techniques for the management of anxiety and fear in pediatric dental practice: a systematic review of randomized controlled trials. Int J Paediatr Dent 2019; 29 (5): 650-668. doi: 10.1111/ipd.12499.

19. De Oliveira RVD, Barbosa AC, Brindeiro D, Gomes RC, Soares FD, Alves S. Student's perceptions about pediatric dental behavior guidance techniques throughout a five-year dental curriculum. Pesqui Bras Odontopediatria Clín. Integr 2015;15(1):143-152.

20. Bimstein E, Azari AF, Sotto JJ, Riley JL. Students' perceptions about pediatric dental behavior guidance in an undergraduate four-year dental curriculum. J Dent Educ 2009; 73(12): 1366- 71.

21. Keskinruzgar A, Ozcan A, Delikan E. Knowledge, attitude and practices of oral and maxillofacial surgeons regarding behavior guidance techniques for managing child patients. CDJ 2018; 21(4): 311- 18.

22. Kawia HM, Mbawalla HS, Kahabuka FK. Application of behavior management techniques for paediatric dental patients by Tanzanian dental practitioners. Open Dent J 2015; 31 (9): 455-61.

23. Wells M, McCarthy BA, Tseng CH, Law CS. Usage of behavior guidance techniques differs by provider and practice characteristics. Pediatr Dent 2018; 40 (3): 201-8.

24. Quiroz-Torres J, Melgar RA. Manejo de conducta no convencional en niños: Hipnosis, musicotera $\neg$ pia, distracción audiovisual y aromaterapia: Revisión sistemática. Rev Estomatol Herediana 2012; 22 (2): 129136.

25. Levy RL, Domoto PK. Current techniques for behavior management: a survey. Pediatr Dent 1979; 1 (3): $160-4$.

26. Wright FA1, McMurray NE, Giebartowski J. Strategies used by dentists in Victoria, Australia, to manage children with anxiety or behavior problems. SDC J Dent Child 1991; 58 (3): 223-8.

27. Strom K, Ronneberg A, Skaare AB, Espelid I, Wilumsen T. Dentists' use of behavioural management techniques and their attitudes towards treating paediatric patients with dental anxiety. Eur Arch Paediatr Dent 2015; 16 (4): 349-55.

28. Radhakrishna S, Srinivasan I, Setty JV, D R MK, Melwani A, Heegde KM. Comparison of three behavior modification techniques for management of anxious children aged 4-8 years. J Dent Anesth Pain Med 2019; 19 (1): 29- 36.

29. McKnight-Hanes C, Myers DR, Dushku JC, Davis HC. The use of behavior management techniques by dentists across practitioner type, age, and geographic region. Pediatr Dent 1993; 15: 267-71

30. Peretz B, Glaicher H, Ram D. Child-Management techniques. Are there differences in the way female and male pediatric dentists in Israel practice? Braz Dent J 2003; 14 (2): 82-86.

Recibido:12/03/2020

Aceptado: 15/05/2020

Correspondencia: Begoña Bartolomé Villar, correo: begona.bartolome@universidadeuropea.es 